\title{
Estímulos domiciliares e características ao nascimento de crianças com síndrome de Down
}

Home stimuli and characteristics at birth of children with Down syndrome Estímulos del hogar y características al nacer de los niños con síndrome de Down

Recebido: 21/08/2019

Aprovado: 01/07/2020

Publicado: 05/08/2020

\author{
Monise Fernandes de Carvalho ${ }^{1}$ \\ Janaine Brandão Lage $^{2}$ \\ Karina Pereira ${ }^{3}$
}

Este é um estudo quantitativo e descritivo realizado entre 2018 a 2019, que tem como objetivo descrever as características de nascimento e estímulos domiciliares de crianças com síndrome de Down. 0 questionário utilizado para coletar informações sobre o ambiente domiciliar da criança foi o Affordances in the Home Environment for Motor Development Self-Report (18 aos 36 meses), através de entrevista, e um questionário de anamnese. Participaram cinco mães e suas crianças com síndrome de Down. As crianças tinham média de 31 meses $( \pm 3,78)$ e, todas realizaram cirurgias. Verificou-se que todas as crianças apresentaram médias e altas oportunidades. $\mathrm{O}$ espaço interior e a variedade de estímulos foram os únicos itens em que todas as crianças receberam pontuação muito boa. A motricidade fina e grossa das crianças foi classificada como fraca. 0 ambiente domiciliar possuía estímulos importantes para o desenvolvimento das crianças. 0 ambiente é importante para o crescimento e desenvolvimento da criança com Down, assim como a participação na estimulação pelos pais e outros adultos ao redor.

Descritores: Meio Ambiente; Síndrome de Down; Jogos e brinquedos.

This is a quantitative and descriptive study carried out between 2018 and 2019. It aims to describe the characteristics of birth and home stimuli of children with Down syndrome. The questionnaire used to collect information about the child's home environment was the Affordances in the Home Environment for Motor Development Self-Report (18 to 36 months), through an interview, and an anamnesis questionnaire. Five mothers and their children with Down syndrome participated. The children had an average age of 31 months $( \pm 3.78)$ and all of them underwent surgery. It was found that all children had medium and high opportunities. The interior space and the variety of stimuli were the only items in which all children received a very good score. The fine and gross motor skills of children were classified as weak. The home environment had important stimuli for children's development. The environment is important for growth and development of the child with Down, as well as participation in stimulation by parents and other adults around.

Descriptors: Environment; Down Syndrome; Play and playthings.

Este es un estudio cuantitativo y descriptivo realizado entre 2018 y 2019 , que tiene por objeto describir las características de nacimiento y los estímulos del hogar de los niños con síndrome de Down. El cuestionario utilizado para recoger información sobre el entorno del hogar del niño fue el Affordances in the Home Environment for Motor Development Self-Report (18 a 36 meses), a través de una entrevista, y un cuestionario de anamnesis. Participaron cinco madres y sus hijos con síndrome de Down. Los niños tenían un promedio de 31 meses $( \pm 3,78)$ y todos ellos realizaron cirugías. Se verificó que todos los niños tenían oportunidades medias y altas. El espacio interior y la variedad de estímulos fueron los únicos elementos en los que todos los niños recibieron una puntuación muy buena. La motricidad fina y gruesa de los niños fue clasificada como débil. El entorno del hogar tenía importantes estímulos para el desarrollo de los niños. El entorno es importante para el crecimiento y desarrollo del niño con Down, así como la participación en el estímulo de los padres y otros adultos de alrededor.

Descriptores: Ambiente; Síndrome de Down; Juego e Implementos de juego.

1. Fisioterapeuta. Uberaba, MG, Brasil. ORCID: 0000-0002-3101-5219 E-mail: mohfernande@hotmail.com

2. Fisioterapeuta. Especialista em Acupuntura. Especialista em Neuropediatria. Mestre em Educação Física. Doutoranda em Ciências da Saúde pela Universidade Federal do Triângulo Mineiro (UFTM). Fisioterapeuta do Centro de Equoterapia da Associação de Pais e Amigos dos Excepcionais (APAE), Uberaba, MG, Brasil. ORCID: 0000-0002-1712-5593 E-mail: ja.bl@terra.com.br

3. Fisioterapeuta. Especialista em Intervenção em Neuropediatria. Mestre e Doutora em Fisioterapia. Professor Associado do Departamento de Fisioterapia e do Programa de Pós-Graduação em Fisioterapia da UFTM, Uberaba, MG, Brasil. 0RCID: 0000-0001-7486-1004 E-mail: karina.pereira@uftm.edu.br 


\section{INTRODUÇÃO}

$\mathbf{0}$ desenvolvimento infantil sofre algumas interferências sociais e ambientais durante sua evolução, devido às características do ambiente domiciliar da criança em relação a quantidade, variedade dos estímulos e participação da família ${ }^{1}$. 0 ambiente em que a criança está inserida pode atuar como facilitador do seu desenvolvimento. E a relação dos pais com a criança é importante ao ajudá-la a ter mais liberdade de movimento, auxiliar a ficar em pé, a falar, a brincar e interagir com outras crianças e adultos ${ }^{2}$.

A família e outros adultos dentro do contexto de ambiente domiciliar são essenciais no desenvolvimento da criança, proporcionando as primeiras experiências de aprendizagem, por meio do ato de brincar $^{3}$. A teoria ecológica, segundo Gibson define as affordances (oportunidades) do ambiente como aquilo que oferece um desafio para a pessoa, ou seja, algo que proporciona as primeiras interações com o ambiente interno e externo do domicílio, como os objetos, as superfícies e as pessoas ${ }^{4,5}$.

0 nascimento de um filho pode exigir adaptação dos pais, ocorrendo mudanças no diaa-dia, ainda mais após o diagnóstico de uma síndrome que representa atraso no desenvolvimento do bebê6. A síndrome de Down, é uma alteração genética e têm com característica a lentidão no desenvolvimento motor e aspectos clínicos como hipotonia muscular, frouxidão ligamentar, hipermobilidade articular, déficit cognitivo e atraso na fala, que podem ser um fator limitante na exploração do ambiente e dos brinquedos devido à dificuldade dos pais em fornecer estímulos de acordo com o interesse da criança7.

A disponibilidade de estímulos como brinquedos, que são fundamentais para o desenvolvimento infantil, como os de pelúcia, carros, animais, puzzles e jogos de quebracabeça, brinquedos de construção de vários tamanhos, caixa de areia, lápis para colorir, livros, caixas de músicas, instrumentos musicais, triciclos, bicicletas e balanços, são o suporte de muitas brincadeiras que incentivam a aquisição de coordenações motoras finas e grossas ${ }^{8}$.

Os desfechos do crescimento infantil são influenciados por fatores de riscos biológicos e sociais como moradia (características do local), relações familiares, escolaridade dos pais e renda familiar ${ }^{9}$. Família com melhores condições financeiras, com maior grau de escolaridade, em que o chefe de família possui uma profissão bem remunerada, são mais propensas a possuir maior variedade de bens e serviços que irão privilegiar os seus filhos ${ }^{10}$. Com isso, as crianças que se encontra em uma situação mais favorável possui uma melhor estimulação. Neste sentido, este relato de casos tem como objetivo descrever as características de nascimento e estímulos domiciliares de crianças com síndrome de Down.

\section{MÉTODO}

Este é um estudo quantitativo e descritivo que foi aprovado pelo Comitê de Ética em Pesquisa (CEP) da UFTM (parecer 2.695.020). A coleta de dados foi realizada por meio de entrevista de dezembro de 2018 a março de 2019 na Universidade Federal do Triângulo Mineiro em Uberaba, Minas Gerais.

Aplicou-se às mães o questionário Affordances in the Home Environment for Motor Development Self-Report (AHEMD-SR - 18 a 36 meses) por meio de entrevistas, durante 20 minutos.

As pontuações foram geradas a partir do programa (AHEMD-SR Calculator VPbeta 1.5.xls) de acordo com as cinco subescalas sendo: espaço interior (espaço para brincar e andar livremente, mais de um tipo de solo, mobília para a criança subir, descer e saltar, quarto de brinquedos e baús para guardar os brinquedos), espaço exterior (seria um jardim ou quintal e pode ter mais de um tipo de solo como: grama, cimento, madeira; superfícies inclinadas, brinquedos para a criança se pendurar, escadas, local destinado para a criança brincar).

A variedade de estimulação (se a criança brinca com outras crianças, os pais brincam com ela, as crianças brincam com outros adultos além dos pais; também se a vestimenta da 
criança permite liberdade de movimento, quanto tempo ela fica em uma região especifica da casa, num parque, livre para andar por toda residência), motricidade fina (será observada a quantidade dos brinquedos que criança possui, variando de zero a mais de 5 e são: fantoches, brinquedos que imitam telefones, material de cozinha, ferramentas, jogos, brinquedos de encaixar, tabuleiros com peças de encaixar, lego, lápis de cor, lápis de cera, papel, tintas, massinha ou argila para moldagem, tesoura sem pontas; brinquedos musicais sendo: guizos, chocalhos, pandeiros e pianinhos) e motricidade grossa (bolas de diferentes tamanhos, cores e materiais, bastões e betes, alvos, cestos, cones, cavalos de pau, patinetes, escorregadores, túneis, triciclos, bicicletas, carros e balanços) que recebem pontuações independentes de acordo com a sua qualidade: muito boa (4), boa (3), fraca (2), muito fraca (1) e ruim (0). Ao final o AHEMD-SR total classifica as oportunidades de estimulação do ambiente domiciliar em "baixa" (ambiente oferece pouca oportunidade), "média" (ambiente razoável) ou "alta" (excelente ambiente) ${ }^{11}$.

\section{RESULTADOS}

Participaram cinco crianças, das quais quatro eram meninas, com média de idade gestacional de 35 semanas $( \pm 2,28)$, média de idade de 31 meses $( \pm 3,78)$, média de peso ao nascer de $2.278 \mathrm{~kg}( \pm 416)$, média do comprimento ao nascer de $42,3 \mathrm{~cm}( \pm 5,21)$ e média do Apgar no quinto minuto de $9( \pm 1,14)$.

Todas as crianças realizaram algum tipo de cirurgia, sendo três crianças submetidas a cirurgias cardíacas e duas do trato gastrointestinal (Tabela 1).

Tabela 1. Caracterização das crianças com síndrome de Down. Uberaba-MG, 2019

\begin{tabular}{cccccccc}
\hline Crianças & $\begin{array}{c}\text { Idade } \\
\text { gestacional }\end{array}$ & Apgar & Peso & Comprimento & Cirurgia & $\begin{array}{c}\text { Tempo de } \\
\text { Internação }\end{array}$ & $\begin{array}{c}\text { Idade } \\
\text { Cronológica }\end{array}$ \\
\hline A & $38 \mathrm{sem}$ & 10 & $2.600 \mathrm{~kg}$ & $48 \mathrm{~cm}$ & Colecistectomia & 2 dias & $36 \mathrm{meses}$ \\
$\mathrm{B}$ & $33 \mathrm{sem}$ & 9 & $1.800 \mathrm{~kg}$ & $42 \mathrm{~cm}$ & Cardíaca & 180 dias & $32 \mathrm{meses}$ \\
C & $35 \mathrm{sem}$ & 7 & $2.200 \mathrm{~kg}$ & $42,5 \mathrm{~cm}$ & Gastroesofágica & 14 dias & 33 meses \\
D & $33 \mathrm{sem}$ & 8 & $1.990 \mathrm{~kg}$ & $45 \mathrm{~cm}$ & Cardíaca & 210 dias & 29 meses \\
E & $37 \mathrm{sem}$ & 9 & $2.800 \mathrm{~kg}$ & $34 \mathrm{~cm}$ & Cardíaca & 21 dias & 26 meses \\
\hline
\end{tabular}

Legenda: sem- semanas, kg- kilogramas, cm- centímetros.

As características familiares estão apresentadas na Tabela 2 segundo o questionário AHEMD-SR. A casa foi o tipo de residência mais comum, grau de escolaridade dos pais prevaleceu o ensino médio, quanto à renda mensal, na maioria das famílias foi acima de $\mathrm{R} \$ 2.500,00$.

Tabela 2. Características familiares das crianças com síndrome de Down, segundo AHEMD-SR. Uberaba - MG, 2019.

\begin{tabular}{lccccc}
\hline Característica da família & Criança A & Criança B & Criança C & Criança D & Criança E \\
\hline Tipo de residência & Apartamento & Casa & Casa & Casa & Casa \\
Quantidade de adultos & 2 & 2 & 1 & 2 & 2 \\
Quantidade de crianças & 2 & 1 & 2 & 2 & 1 \\
Escolaridade - Pai & Superior & Superior & Médio & Médio & Médio \\
Escolaridade - Mãe & Superior & Médio & Médio & Médio & Médio \\
Renda mensal (reais) & $\mathrm{R} \$ 5.000$ ou & $\mathrm{R} \$ 5.000$ ou & $\mathrm{R} \$ 2.500 \mathrm{a}$ & $\mathrm{R} \$ 2.500 \mathrm{a}$ & $\mathrm{R} \$ 1.000 \mathrm{a}$ \\
& mais & mais & $\mathrm{R} \$ 3.500$ & $\mathrm{R} \$ 3.500$ & $\mathrm{R} \$ 1.500$ \\
\hline
\end{tabular}

A Figura 1 mostra a pontuação obtida pelas crianças em cada uma das subescalas do AHEMD-SR. 0 espaço interior e a variedade de estímulos foram os únicos itens em que todas as crianças receberam pontuação 4 (muito boa). No espaço exterior, apenas uma criança apresentou pontuação 4. Na motricidade grossa, quatro crianças tiveram pontuação baixa.

As crianças D e E apresentaram as menores pontuações na caracterização do seu ambiente domiciliar (fraca e/ou muito fraca) nos itens ambiente externo, motricidade fina e grossa. No entanto, na pontuação total do AHEMD-SR foram classificadas com razoáveis oportunidades, isso mostra a importância de analisar cada item para que a causa das 
dificuldades possa ser identificada e readequada de acordo com as condições financeiras das famílias.

Figura 1. Caracterização do perfil da criança no ambiente domiciliar segundo a AHEMD-SR. Uberaba-MG, 2019.

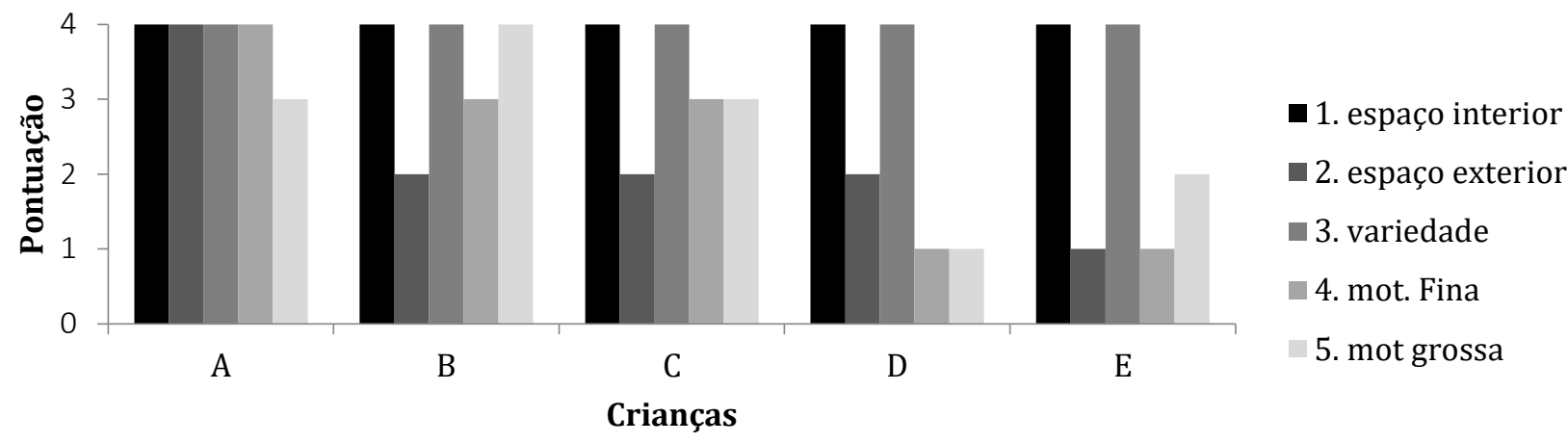

Legenda: As letras A, B, C, D e E representam as cinco crianças do estudo.

Na Tabela 3 observa-se que todas as crianças apresentaram elevada quantidade de brinquedos de motricidade fina, sendo maior que a de brinquedos de motricidade grossa.

Tabela 3. Quantidade dos materiais de motricidade fina e grossa (AHEMD-SR). Uberaba-MG, 2019.

\begin{tabular}{|c|c|c|c|c|c|}
\hline AHEMD-SR & Criança A & Criança B & Criança C & Criança D & Criança E \\
\hline & \multicolumn{5}{|c|}{ MOTRICIDADE FINA } \\
\hline Bonecos de faz de conta & $\geq 5$ & $\geq 5$ & $\geq 5$ & $\geq 5$ & $\geq 5$ \\
\hline Puzzles & $\geq 5$ & $\geq 5$ & $\geq 5$ & 3 ou 4 & $\geq 5$ \\
\hline Jogos & $\geq 5$ & $\geq 5$ & $\geq 5$ & 1 ou 2 & 3 ou 4 \\
\hline Materiais de construção & $\geq 5$ & 1 ou 2 & 3 ou 4 & 1 ou 2 & 1 ou 2 \\
\hline Brinquedos educacionais & $\geq 5$ & $\geq 5$ & $\geq 5$ & 1 ou 2 & $\geq 5$ \\
\hline \multirow[t]{2}{*}{ Outros } & 3 ou 4 & $\geq 5$ & $\geq 5$ & 1 ou 2 & 1 ou 2 \\
\hline & \multicolumn{5}{|c|}{ MOTRICIDADE GROSSA } \\
\hline Materiais Musicais & $\geq 5$ & $\geq 5$ & $\geq 5$ & 3 ou 4 & $\geq 5$ \\
\hline Materiais manipulativos & 3 ou 4 & $\geq 5$ & 3 ou 4 & 1 ou 2 & 1 ou 2 \\
\hline Materiais locomotores & $\geq 5$ & $\geq 5$ & $\geq 5$ & 1 ou 2 & 3 ou 4 \\
\hline Materiais exploração global & 1 ou 2 & 1 ou 2 & 3 ou 4 & 1 ou 2 & 1 ou 2 \\
\hline
\end{tabular}

A Tabela 4 mostra a pontuação do AHEMD total de cada criança, sendo possível observar que a pontuação variou de média a alta e o ambiente de razoável a excelente.

Tabela 4. Classificação de cada criança no escore total do AHEMD-SR. Uberaba- MG, 2019.

\begin{tabular}{cccc}
\hline Crianças & Escore & Pontuação & Classificação das oportunidades de estímulos \\
\hline A & 19 & Alta & Ambiente excelente \\
B & 17 & Alta & Ambiente excelente \\
C & 16 & Média & Ambiente razoável \\
D & 12 & Média & Ambiente razoável \\
E & 12 & Média & Ambiente razoável \\
\hline
\end{tabular}

\section{DISCUSSÃO}

É entendido que fatores como o tipo de domicílio em que a criança habita pode influenciar nos estímulos oferecidos a ela, desde os espaços existentes como: ambiente interno e externo, a presença ou não de irmãos, os adultos e crianças que vivem no ambiente da criança, o grau de escolaridade dos pais, o nível socioeconômico, entre outros fatores que intervêm nas oportunidades de forma a conferir desafios ao indivíduo em desenvolvimento ${ }^{12}$.

A criança A nasceu com peso adequado para a idade gestacional (AIG) e a termo. Sobre o espaço exterior, sendo um terraço, a criança tem contato com superfícies inclinada para descer, subir ou saltar e escadas, tem oportunidade de brincar no parque infantil do seu condomínio. 0 
espaço interno mostrou-se suficiente para brincar, tem móveis para se pendurar com segurança e um quarto de brinquedos e brincadeiras. Sobre as atividades diárias, a criança A não brinca todos os dias com outras crianças, no entanto, regularmente brinca com outros adultos além dos pais. Os pais permitem que ela escolha suas brincadeiras, ensinando a reconhecer as diferentes partes do corpo. A mãe relata que a residência é muito pequena. Em relação aos brinquedos de motricidade grossa, a criança teve pouca quantidade em materiais de exploração global e teve uma pontuação do AHEMD-SR total de 19 pontos. A variedade de estimulação (brincadeiras com outras crianças, escolha de seus brinquedos, estímulo de brincar por parte dos pais, roupas confortáveis para brincar e tempo que permanece em determinados ambientes) pode ser um preditor significativo para crescimento ${ }^{13}$.

A criança B nasceu com peso adequado para a idade gestacional e pré-termo. Em relação ao espaço exterior, conta com rampas de inclinações variadas e um local para as crianças brincarem, como exemplo um parque infantil. 0 espaço interior é suficiente para brincar e andar; não tem mais de um tipo de solo sendo, piso frio em todos os ambientes, e também não existe um quarto de brinquedos, mas guarda-os dentro de baús ou gavetas. Sobre as atividades diárias, a criança B brinca com outras crianças e outros adultos além de, quase sempre, os pais. Os pais brincam com a criança ensinando e estimulando a criança com voz de comando para a criança correr, andar, engatinhar e parar, a criança não escolhe sempre os brinquedos que quer para brincar. Sobre passear no parque infantil, ela frequenta quase sempre e a casa é considerada de tamanho razoável, moderado. A criança teve pouca quantidade em brinquedo de motricidade fina que são os materiais de construção e motricidade grossa, que são os materiais de exploração global. O AHEMD-SR total foi de 17 pontos. Um estudo realizado no Rio Grande do Sul (SINPRO), na cidade de Santa Maria - RS realizado com responsáveis de crianças típicas entre 18 aos 42 meses, mostra como os ambientes domiciliares possuem uma fragilidade em relação aos materiais oferecidos para as crianças, o quanto isso afeta diretamente a motricidade na infância ${ }^{3}$.

A criança C nasceu com o peso adequado para a idade gestacional e pré-termo. Realizou cirurgia gastroesofágica e ficou hospitalizada durante 14 dias. Sobre o espaço exterior tem mais de um tipo de solo (grama e cimento), não possui superfícies inclinadas, tem brinquedo para pendurar-se, não possui tem escadas. No espaço interior, a casa não tem espaço suficiente, possui um carpete no solo para a criança cair com segurança, e também existe mobília para subir, descer e saltar, não possui quarto de brinquedo, mas sim baús para guardá-los. Quanto às atividades diárias, brinca com outros adultos e outras crianças, pode escolher os brinquedos para brincar e os pais encorajam e estimulam a criança nas brincadeiras. No parque infantil fica durante muito tempo, quase sempre é livre para andar por toda casa e a casa é considerada pequena. A criança teve uma quantidade média de brinquedo tanto em motricidade fina quanto em motricidade grossa, o AHEMD-SR total foi de 16 pontos, classificada como média. Um trabalho descreve que uma residência classificada como média proporciona oportunidades razoáveis para o desenvolvimento motor da criança ${ }^{14}$.

A criança D quando nasceu teve peso adequado para a idade gestacional (AIG) e prétermo. Realizou cirurgia cardíaca e o tempo de internação foi de 210 dias. Em relação ao espaço exterior, não tem escada, não possui solos diferentes e não tem brinquedos em uma área simulando um parque infantil, e o espaço não possui rampas. No ambiente interior tem espaço suficiente para brincar e andar, mobília para pendurar-se e tem quarto de brinquedos e baús. Nas atividades diárias, a criança $\mathrm{D}$ brinca com outras crianças e adultos além dos pais. Os pais brincam com a criança permitindo que ela escolha seus brinquedos, ensinam sobre partes do corpo humano. Em um parque infantil ela permanece durante pouco tempo, livre para andar na casa muito tempo e o tamanho da casa é considerado amplo grande.

A criança $C$ teve pequena quantidade de brinquedo na subescala de motricidade fina nos itens de jogos, materiais de construção, brinquedos educacionais e outros. Na subescala de motricidade grossa teve pouca quantidade em materiais manipulativos, materiais locomotores, 
materiais de exploração global; AHEMD-SR total de 12 pontos. Realizado em Juazeiro do Norte, município localizado na região Sul do estado do Ceará, o estudo mostra que ao utilizar o mesmo questionário AHEMD-SR (18 e 36 meses) com responsáveis de crianças com idade entre 33 e 38 semanas (como neste relato), evidenciou que a situação de escassez de oportunidade de estímulos ambientais poderá comprometer o desenvolvimento motor das crianças, visto que, a disponibilidade de materiais e objetos estimulantes são importantes no desenvolvimento15.

A criança E nasceu pré-termo, peso adequado para a idade gestacional e realizou cirurgia cardíaca, com tempo de internação de 21 dias. A casa não possui um ambiente externo. 0 ambiente interior é suficiente para a criança brincar e andar, dentro da casa não tem vários tipos solo, tem mobília para se pendurar com segurança, não tem escadas, possui um quarto só de brinquedos, baús e gavetas. Sobre as atividades diárias a criança não brinca todos os dias com outras crianças e brinca regularmente com outros adultos além dos pais, a criança escolhe os brinquedos para brincar, e os pais brincam e estimulam a criança.

A criança E quase nunca vai ao parque, fica livre em casa para andar. A criança teve pouca quantidade de brinquedos em motricidade fina nos itens de materiais de construção e outros e motricidade grossa nos itens de materiais manipulativos, materiais de exploração global; AHEMD -SR total foi de 12 pontos. 0 espaço externo foi a variável que apresentou maior inadequação, o mesmo foi observado no estudo, que avaliou o ambiente domiciliar de crianças típicas de 32 a 48 meses de uma escola municipal de Campina Grande do Sul-PR, verificandose que o espaço exterior da maioria das residências analisadas não oferecia oportunidades suficientes para o desenvolvimento das crianças ${ }^{13}$.

Uma pesquisa efetuada com lactentes que possuem síndrome Down, de idades entre 3 e 18 meses, residentes na região do Triângulo Mineiro (MG), demonstrou que as limitações da síndrome interferem no desempenho motor e também que existem precárias oportunidades domiciliares para esses lactentes. Mesmo com as limitações, as crianças com síndrome de Down com mais affordances proporcionadas pelos pais podem apresentar uma socialização mais fácil com o ambiente e uma melhor interação com brinquedos, desde que sejam estimuladas e tenham essas oportunidades ${ }^{2}$.

\section{CONCLUSÃo}

0 ambiente domiciliar e as características ao nascimento das crianças são fundamentais para o desenvolvimento, as crianças nasceram com algumas limitações como a prematuridade e, em seguida, realizaram cirurgias, com isso, é muito importante a estimulação.

0 ambiente proporcionará um bom crescimento, assim como os pais e outros adultos que possuem um papel importante na vida dessas. Com isso, é importante que novos estudos sejam feitos, para sempre auxiliar na vida das crianças e dos pais que tanto precisam de ajuda, visando melhorar o desenvolvimento, já que as mesmas possuem síndrome de Down, e possuem certas características limitantes.

O estudo tem como limitação o reduzido número de crianças pesquisadas, mas mostra a realidade de crianças com Down que pode ser idêntica as outras localidades.

\section{REFERÊNCIAS}

1. Eickmann SH. Desenvolvimento infantil: fatores determinantes e impacto de um programa de estimulação psicossocial [tese]. Recife: Universidade Federal de Pernambuco; 2003.

2. Knychala NAG, Oliveira EA, Araújo LB, Azevedo VMGO. Influência do ambiente domiciliar no desenvolvimento motor de lactentes com síndrome de Down. Fisioter Pesqui. [Internet]. 2018 [citado em 02 jul 2020]; 25(2):202-8. DOI: http://dx.doi.org/10.1590/18092950/17006925022018

3. Soares ES, Flores FS, Katzer JI, Valentini NC, Corazza ST, Copetti F. Análise das oportunidades de estimulação motora em ambientes domiciliares na região central do Rio Grande do Sul. Rev 
Bras Educ Fís Esp. [Internet]. 2015 [citado em 02 jul 2020]; 29(2):279-88. DOI: http://dx.doi.org/10.1590/1807-55092015000200279

4. Iltus S. Significance of home environments as proxy indicators for early childhood care and education. Background paper prepared for the Education for all Global Monitoring Report 2007, Strong foundations: early childhood care and educations [Internet]. [Paris: UNESCO]; 2006 [citado em 02 jul 2020]. (2007/ED/EFA/MRT/PI/16). Disponível em: https://pdfs.semanticscholar.org/4437/ec766798fb8f43d2a9449736bdbd5640c771.pdf

5. Harold SJ. Gibson's "Affordances": evolution of a pivotal concept. J Sci Psychol. [Internet]. 2008 [citado em 02 jul 2020]; 34-45. Disponível em: https://www.psyencelab.com/uploads/5/4/6/5/54658091/gibsons_affordances_evolution_ of_a_pivotal_concept.pdf

6. Bradt JO. Tornando-se pais: famílias com filhos pequenos. In: Carter B, McGoldrick M. As mudanças no ciclo de vida familiar: uma estrutura para a terapia familiar. Trad. Veronese MAV. Porto Alegre: Artes Médicas; 1995. p. 206-22

7. Pereira K, Basso RP, Lindquist ARR, Silva LGP, Tudella E. Infants with Down syndrome: percentage and age for acquisition of gross motor skills. Res Dev Disabil. [Internet]. 2013 [citado em 02 jul 2020]; 34(3):894-901. DOI: 10.1016/j.ridd.2012.11.021

8. Vygotsky LS, Luria AR, Leontiev AN. Linguagem, desenvolvimento e aprendizagem. São Paulo: Ícone; 1998.

9. Rodrigues LM. A criança e o brincar [monografia]. Rio de Janeiro: Universidade Federal Rural do Rio de Janeiro; 2009.

10. Tong S, Baghurst P, Vimpani G, McMichael A. Socioeconomic position, maternal IQ, home environment and cognitive development. J Pediatr. [Internet] 2007 [citado em 02 jul 2020]; 151(3)284-8. DOI: 10.1016/j.jpeds.2007.03.020

11. Rodrigues LP, Saraiva L, Gabbard C. Development and constructural validation of an inventory for assessing the home environment for motor development. Res Quar Exerc Sport. [Internet]. 2005 [citado em 02 jul 2020]; 76(2):140-8. DOI: http://dx.doi.org/10.1080/02701367.2005.10599276

12. Silva TR, Gontijo CS. A família e o desenvolvimento infantil sob a ótica da gestalt-terapia. Rev IGT Rede [Internet]. 2016 [citado em 02 jul 2020]; 13(24):15-36. Disponível em: http://pepsic.bvsalud.org/pdf/igt/v13n24/v13n24a03.pdf

13. Silva WR, Lisboa T, Ferrari EP, Freitas KTD, Cardoso FL, Motta NFA, et al. Opportunities for motor stimulation in the home environment of children. J Hum Growth Dev. [Internet]. 2017 [citado em 02 jul 2020]; 27(1):84-90. DOI: http://dx.doi.org/10.7322/jhgd.127659

14. Rodrigues LP, Gabbard C. A invariância do gênero na estrutura multidimensional do AHEMD (Affordances in the Home Environment for Motor Development). In: Catela D, Barreiros J, editores. Estudos em desenvolvimento motor da criança. Rio Maior, Portugal: Edições ESDRRM; 2008. p. 151-8.

15. Nobre FSS, Costa CLA, Oliveira DL, Cabral DA, Nobre GC, Caçola P. Análise das oportunidades para o desenvolvimento motor (affordances) em ambientes domésticos no Ceará - Brasil. Rev Bras Crescimento Desenvolv Hum. [Internet]. 2009 [citado em 02 jul 2020]; 19(1):9-18. Disponível em: http://pepsic.bvsalud.org/pdf/rbcdh/v19n1/02.pdf 


\section{CONTRIBUIÇÕES}

Monise Fernandes de Carvalho contribuiu na coleta e interpretação dos dados e, redação. Janaine Brandão Lage colaborou na interpretação dos dados e, redação. Karina Pereira atuou na interpretação dos dados e revisão.

\section{Como citar este artigo (Vancouver)}

Carvalho MF, Lage JB, Pereira K. Estímulos domiciliares e características ao nascimento de crianças com síndrome de Down. REFACS [Internet]. 2020 [citado em inserir dia, mês e ano de acesso]; 8(Supl. 2):746-753. Disponível em: inserir link de acesso. DOI: inserir link do DOI.

\section{Como citar este artigo (ABNT)}

CARVALHO, M. F.; LAGE, J. B.; PEREIRA, K. Estímulos domiciliares e características ao nascimento de crianças com síndrome de Down. REFACS, Uberaba, MG, v. 8, p. 746-753, 2020. Supl. 2. Disponível em: inserir link de acesso. Acesso em: inserir dia, mês e ano de acesso. DOI: inserir link do DOI.

\section{Como citar este artigo (APA)}

Carvalho, M.F., Lage, J.B., \& Pereira, K. (2020). Estímulos domiciliares e características ao nascimento de crianças com síndrome de Down. REFACS, 8(Supl. 2), 746-753. Recuperado em inserir dia, mês e ano de acesso de inserir link de acesso. DOI: inserir link do DOI. 